\title{
The Prognostic Effects of Clinicopathological Features on Rectal Cancer Patients undergoing Neoadjuvant Chemoradiotherapy
}

\author{
Alaettin Arslan ${ }^{1}$, Ebru Akay², Gamze Turk ${ }^{3}$ and Saliha Karagoz Eren ${ }^{4}$ \\ ${ }^{1}$ Department of Radiation Oncology, Kayseri City Hospital, Kayseri, Turkey \\ ${ }^{2}$ Department of Pathology, Kayseri City Hospital, Kayseri, Turkey \\ ${ }^{3}$ Department of Radiology, Kayseri City Hospital, Kayseri, Turkey \\ ${ }^{4}$ Department of General Surgery, Kayseri City Hospital, Kayseri, Turkey
}

\begin{abstract}
Objective: To investigate the effects of clinicopathological features on disease-free survival (DFS) and overall survival (OS) in in-patients with local advanced rectal cancer (LARC) who received neoadjuvant chemoradiotherapy (nCRT).

Study Design: Observational study.

Place and Duration of Study: Clinic of Radiation Oncology, Kayseri Training and Research Hospital and Kayseri City Hospital, Turkey, between January 2014 and June 2019.

Methodology: The pre-nCRT, post-nCRT, and postoperative imaging methods of 51 patients, who were operated upon, were examined. Radiological images (CT and MRI) of the patients were reviewed using the hospital's PACS system. Pathology reports and preparations were re-evaluated, and TNM staging and the pathological tumour regression grade (pTRG) were graded according to the American Joint Committee on Cancer's (AJCC) 2018 version. For the descriptive statistics of the data, the mean, standard deviation, lowest-highest median, frequency, and ratio values were used. Cox regression (univariate-multivariate analysis) and Kaplan-Meier curves were used for survival analysis.

Results: In the univariate model, the postoperative pathological T and N stages (ypT and ypN), pathological stage, positive lymph node count (pLN+, pathological sampling) and lymphovascular invasion (LVI) positivity had a significant effect $(p<0.05)$ on DFS. In the multivariate reduced model, a significant independent $(p<0.05)$ effect of the ypT and $p L N+$ number was observed on DFS. In the univariate model, the pathological tumour diameter after nCRT, the ypT, perineural invasion (PNI) positivity, and relapse presence had a significant effect $(p<0.05)$ on OS. In the multivariate reduced model, a significant independent $(p<0.05)$ effect of recurrence was observed on OS.

Conclusion: LVI, the ypTN stage, and the pLN+ number affected the disease-free survival, while the residual tumour diameter after nCRT, ypT stage, and PNI affected the overall survival. The predicted DFS time decreased as the ypT stage increased and the predicted OS time decreased as the recurrence increased.
\end{abstract}

Key Words: Rectal cancer, Neoadjuvant chemoradiotherapy, Disease-free survival, Overall survival.

How to cite this article: Arslan A, Akay E, Turk G, Eren SK. The Prognostic Effects of Clinicopathological Features on Rectal Cancer Patients undergoing Neoadjuvant Chemoradiotherapy. J Coll Physicians Surg Pak 2021; 31(04):422-428.

\section{INTRODUCTION}

Treatment of rectal cancer requires a multidisciplinary approach. The essential starting criterion for this is clinically and radiologically correct staging. Anorectal ultrasonography, computed tomography (CT), and magnetic resonance imaging (MRI) are used in the clinical and radiological staging of rectal cancer.

Correspondence to: Dr. Alaettin Arslan, Department of Radiation Oncology, Kayseri City Hospital, Kayseri,

Turkey

E-mail: alaettin.arslan@gmail.com

Received: December 30, 2020; Revised: February 24, 2021;

Accepted: March 10, 2021

DOI: https://doi.org/10.29271/jcpsp.2021.04.422
Their sensitivity and specificity differ in showing perirectal tissue invasion and lymph node (LN) involvement. ${ }^{1}$ Potentially, the primary treatment for curative rectal cancer is surgery. The definition of radial microscopic lymphatic spread within the mesorectum led to the use of total mesorectal excision (TME). Local control rates have increased significantly with this type of surgery. However, even with TME, the pathological node-positive disease's local failure rate is $21 \%$, and adjuvant chemoradiotherapy (CRT) is still required. ${ }^{2}$ For local advanced rectal cancer (LARC), thestandard treatment is neoadjuvant chemoradiotherapy (nCRT), followed by TME. Responses to CRT vary, but the rates of complete pathological response ( $p C R)$ have been reported diffeently in various studies. About half of patients with rectal cancer receive $\mathrm{nCRT}$ due to LARC; the aim is to shrink the tumour. With $\mathrm{nCRT}$, the surgical margin safety rate may 
increase, and the level of radical operation originally planned with an MRI may decrease. ${ }^{3}$ Further, in a small group of patients, the option of surgery may be postponed in patients who have no evidence of clinical or radiological tumours. ${ }^{4}$

The pathological tumour regression grade (pTRG) is a system used for the histological evaluation of tumour response to nCRT. Studies on the use of the PTRG in determining the prognosis of LARC were carried out., ${ }^{5,6}$ The American Joint Committee on Cancer's (AJCC) pTRG is one of the treatment response systems used in rectal cancer, and it is rated $0-3 .^{7}$

Many factors that determine the prognosis in LARC patients receiving nCRT have been investigated. Although the degree of pathological response is considered the most important prognostic factor, the TNM, pTRG, and peripheral resection margin are still major prognostic factors according to some investigators. ${ }^{6,8}$ The prognostic value of the postoperative pathological T stage (ypT) has often been questioned. However, it is still not very reliable to evaluate the prognosis of LARC using the ypTN category alone. ${ }^{9}$ Additionally, perineural invasion (PNI) and lymphovascular invasion (LVI) have a negative impact on the oncological outcome of rectal cancer. ${ }^{10}$

The aim of this study was to investigate the effect on diseasefree survival (DFS) and overall survival (OS) in LARC patients receiving $n C R T$ by using their clinical information.

\section{METHODOLOGY}

The study was conducted with the approval of the Non-interventional Clinical Research Ethics Committee of Erciyes University's Faculty of Medicine (Protocol No. 2020/62). Between 2014 and 2019 , one hundred and twenty-two patients treated for rectal cancer were detected in Kayseri Training and Research Hospital and Kayseri City Hospital. Fifty of these patients were treated with adjuvant CRT. The majority of the patients undergoing adjuvant CRT comprised of patients who presented to this centre after being operated upon at other centres. The remaining 72 patients received nCRT. All of these patients had a cT3-4, N0, or any N+ radiological stage. The hospital registration system's archive files were scanned. In the radiotherapy (RT) planning system, the RT dose, duration, and technique of radiotherapy were assessed. The regime they underwent for simultaneous chemotherapy was determined. Twenty-one patients, who were not operated upon for various reasons (almost all of which were because of the patients' preferences) after nCRT, were excluded from the study. Fifty-one patients, with a cT3-4, N0, or any $\mathrm{N}+$ radiological stage at the time of diagnosis were included in the study. All of these patients were those who had completed nCRT, were operated upon, and had regular follow-ups. The pre-nCRT, post-nCRT, and postoperative images of the patients were examined. The radiological images of the patients were analysed retrospectively using the hospital's PACS system. A magnetic resonance imaging (MRI) examination had been performed on the sagittal, axial, and coronal planes by applying a high resolution, two-dimensional, T2weighted, fast spin-echo sequence. The protocol also included a wide, non-contrasting, T1-weighted sequence to evaluate pelvic lymph nodes and pelvic bone marrow. The studied clinical vari- ables included location of the tumour in the rectum, time to start treatment after diagnosis, RT technique applied, time to operation after $\mathrm{nCRT}$; and pathological variables included pathological tumour diameter after nCRT, pathological stage, ypT stage, ypN stage, pTRG, positive lymph node count [pLN+, pathological sampling] number, LVI, and PNI. Pathology reports and preparations were reassessed, and TNM staging and the PTRG were determined according to the AJCC's 2018 version. The starting point for calculating DFS and OS was determined as the patients' first biopsy date.

IBM SPSS version 25.0 (SPSS Inc., IL, USA) was used for statistical comparison. For the descriptive statistics of the data, the mean, standard deviation, lowest-highest median, frequency, and ratio values were used. Cox regression (univariate-multivariate analysis) and Kaplan-Meier curves were used for survival analysis. A p-value of $<0.05$ was considered significant.

\section{RESULTS}

The characteristics of the patients are given in Table I. In Table II, the imaging, $\mathrm{nCRT}$, and pathology information of the patients are given. Four fields, namely 3D-CRT and 5-7 fields for IMRT, as well as double-arc VMAT plans, were for radiotherapy. All patients received a total of 45 Gy $\mathrm{RT}$, with a $180 \mathrm{cGy} /$ day $\times 25$ fraction. Capecitabine was given simultaneously with RT at $825 \mathrm{mg} / \mathrm{m}^{2}$ in two equal doses per day for five weeks. Pathological TNM staging and the PTRG were performed according to the AJCC's 8th version. A pTRG of $0-1$ was included in the group that responded well and 2-3 in the group that responded poorly. Only one patient had pCR (apTRG of 0 ).

In the univariate model, the post-CRT radiological LN+ $(p=$ $0.026)$, pathological tumour diameter after $\mathrm{nCRT}(p=0.006)$, pathological stage $(p=0.031)$, ypTstage $(p=0.006)$, ypN stage ( $p$ $=0.027), \operatorname{pLN}+$ number $(p=0.011)$, and LVI positivity $(p=0.039)$ had a significant effect on DFS. In the multivariate reduced model, a significant independent effect of the ypT stage $(p=0.009)$ and pLN + number $(p=0.004)$ was observed on DFS. When the 3D-CRT standard RT technique was accepted, the IMRT technique had a significant independent effect on DFS time compared to the VMAT technique $(p=0.005)$ (Table III a).

In the group with a residual tumor after nCRT of $>2 \mathrm{~cm} \mathrm{(49.6}$ months [95\% confidence interval $(\mathrm{Cl}): 37.5-61.6]$ ), the predicted DFS time was significantly shorter $(p=0.006)$ than the group with a tumour diameter of $\leq 2 \mathrm{~cm}(71.3$ months [95\% Cl: 66.1-76.5], Figure 1a).

The predicted DFS time in the ypT stage 4 group (22.8 months [95\% Cl: 11.4-34.3]), compared to the group with ypT stage 3 (54.3 months [95\% Cl: 42.7-65.8]), was shorter. However, no statistically significant difference was found ( $p=0.226$, Figure 1b).

In the univariate model, the pathological tumor diameter after nCRT $(p=0.049)$, ypT stage $(p=0.034)$, PNI positivity ( $p=$ $0.041)$, and recurrence ( $p=0.006)$ presence had a significant effect on OS. In the multivariate reduced model, a significant independent effect of recurrence $(p=0.006)$ was observed on os (Table IIIb). 
Table I: The characteristics of the patients.

\begin{tabular}{|c|c|c|c|c|c|c|c|c|}
\hline & & \multicolumn{3}{|c|}{ Min-Max } & \multirow{2}{*}{\begin{tabular}{|l|} 
Median \\
62.0 \\
\end{tabular}} & \multicolumn{3}{|c|}{$\begin{array}{c}\text { Average } \pm S D / n \\
(\%)\end{array}$} \\
\hline \multicolumn{2}{|l|}{ Age at diagnosis in years } & 26 & - & 79 & & 59.9 & \pm & 11.0 \\
\hline \multirow{2}{*}{ Gender } & Female & & & & & 22 & & $43.1 \%$ \\
\hline & Male & & & & & 29 & & $56.9 \%$ \\
\hline \multirow{3}{*}{ Location of the tumour in rectum from anal verge in $(\mathrm{cm})$} & $0-5$ (lower) & & & & & 23 & & $45.1 \%$ \\
\hline & 5-10 (middle) & & & & & 26 & & $51.0 \%$ \\
\hline & 10-15 (upper) & & & & & 2 & & $3.9 \%$ \\
\hline \multicolumn{2}{|l|}{ Pathological tumour diameter after nCRT (mm) } & 0 & - & 80 & 20.0 & 23.5 & \pm & 18.7 \\
\hline \multicolumn{2}{|l|}{ pLN+ number } & 0 & - & 13 & 0.0 & 0.9 & \pm & 2.1 \\
\hline \multirow{2}{*}{ Recurrence } & $(-)$ & & & & & 41 & & $80.4 \%$ \\
\hline & $(+)$ & & & & & 10 & & $19.6 \%$ \\
\hline \multirow{2}{*}{ Death } & $(-)$ & & & & & 45 & & $88.2 \%$ \\
\hline & $(+)$ & & & & & 6 & & $11.8 \%$ \\
\hline \multicolumn{2}{|l|}{ Follow-up time (months) } & 4 & - & 74 & 33.0 & 35.9 & \pm & 19.4 \\
\hline
\end{tabular}

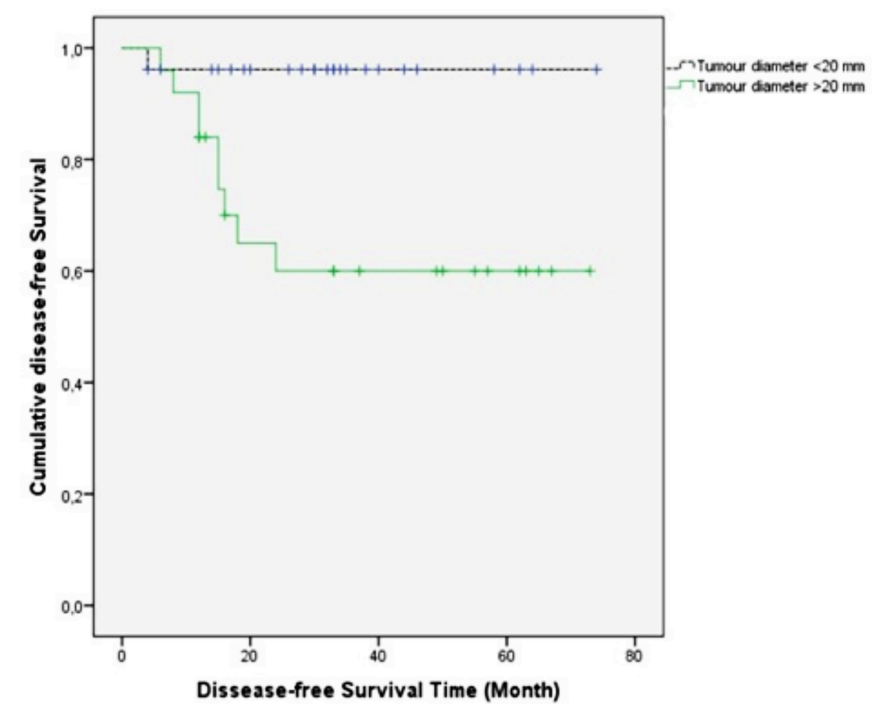

Figure 1a: The effect of pathological tumor diameter after nCRT on DFS (Kaplan-Meier).

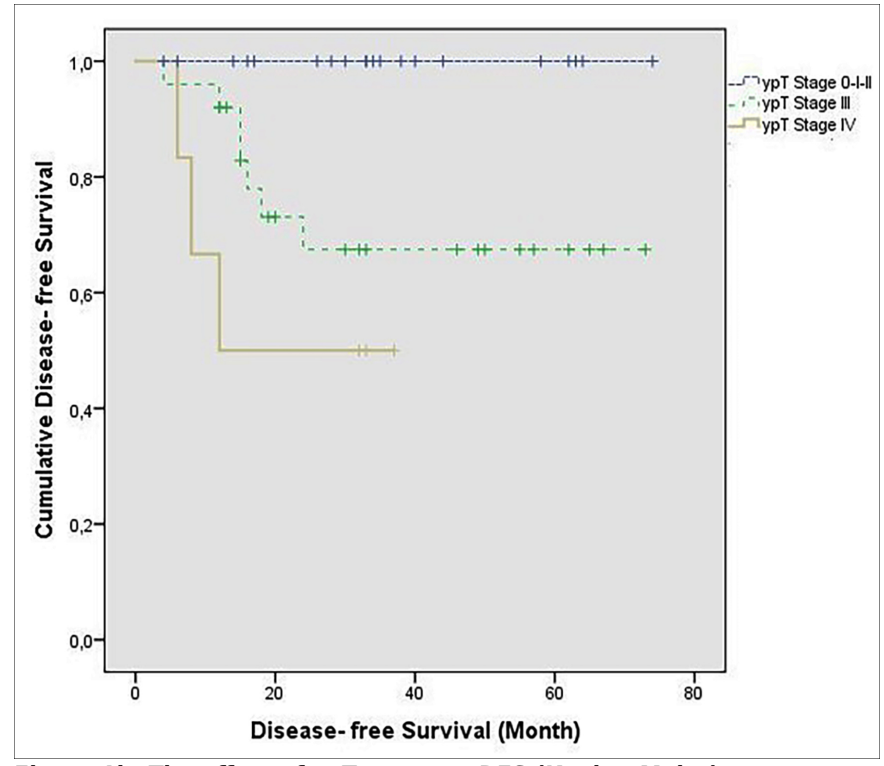

Figure 1b: The effect of ypT stage on DFS (Kaplan-Meier).
The predicted OS in the recurrent group (42.1 months [95\% Cl: 26.7-57.5]) was significantly shorter $(p<0.001)$ than in the non-recurrence group ( 72.4 months [ $95 \% \mathrm{Cl}$ : 69.3-75.5]) (Figure 2).

\section{DISCUSSION}

Although adjuvant therapy can also be effective in the treatment of LARC, neoadjuvant therapy has emerged as the standard. nCRT is the standard treatment for patients with CT3-4NO or any T N+ disease. ${ }^{11}$ Neoadjuvant therapy is associated with the potential for tumour reduction, increased resectability and tolerance, and extended sphincter preservation options in the distal rectum. Studies from Europe have shown that appropriate neoadjuvant pre-operative RT leads to both local control (LC) and OS increase, and these results have a significant impact on the current management of the disease. ${ }^{12,13}$

The Mandard, Rödel, Dworak, and AJCC scoring systems are the most used systems for the PTRG. ${ }^{7,12,14,15}$ In one study, using the AJCC PTRG scoring system, it was stated that the category of PTNM combined with AJCC TRG could more accurately evaluate the prognosis of patients with LARC and determine the subgroup of patients with the worst prognosis and highest risk of distant metastasis. Thus, adjuvant therapy and patient follow-ups were emphasised as being of clinical importance during orientation. ${ }^{9}$ In the study in which 4 pTRG scoring systems were compared, DFS probabilities were estimated using the Kaplan-Meier method. It was emphasised that all TRG systems predicted recurrence. The AJCC system predicted recurrence more precisely than the three-stage Mandard ( $p=0.002$ ) or Dworak/Rödel ( $p=$ 0.006 ) systems and had a higher compatibility index than the MSKCC, although it was not statistically significant ( $p=$ 0.068 ). As a result, when classifying rectal cancer's response to $\mathrm{nCRT}$, it was stated that the AJCC PTRG scoring system is the correct method and should be accepted as standard. ${ }^{16}$ 
Table II: The pre- and post-CRT imaging and treatment, and the postoperative pathology information of the patients.

\begin{tabular}{|c|c|c|c|}
\hline & & & $\begin{array}{l}\text { gge } \pm \text { SD } / n \\
(\%)\end{array}$ \\
\hline \multirow{2}{*}{ Radiological LN+ (pre-CRT) } & $(-)$ & 8 & $15.7 \%$ \\
\hline & $(+)$ & 43 & $84.3 \%$ \\
\hline \multirow{2}{*}{$\begin{array}{l}\text { Radiological LN+ (post- } \\
\text { CRT) }\end{array}$} & $(-)$ & 33 & $64.7 \%$ \\
\hline & $(+)$ & 18 & $35.3 \%$ \\
\hline \multirow{3}{*}{ RT technique } & 3D-CRT & 32 & $62.7 \%$ \\
\hline & IMRT & 13 & $25.5 \%$ \\
\hline & VMAT & 6 & $11.8 \%$ \\
\hline \multirow{2}{*}{$\begin{array}{l}\text { Time between diagnosis } \\
\text { and } \mathrm{nCRT}\end{array}$} & $\leq 3$ weeks & 25 & $49.0 \%$ \\
\hline & $>3$ weeks & 26 & $51.0 \%$ \\
\hline \multirow{2}{*}{$\begin{array}{l}\text { Time between nCRT and } \\
\text { surgery }\end{array}$} & $\leq 8$ weeks & 22 & $43.1 \%$ \\
\hline & >8 weeks & 29 & $56.9 \%$ \\
\hline Chemotherapy agent & Capecitabine & 51 & $100.0 \%$ \\
\hline \multirow{2}{*}{ Histopathology } & Adenocarcinoma & 44 & $86.3 \%$ \\
\hline & Mucinous carcinoma & 7 & $13.7 \%$ \\
\hline \multirow{4}{*}{ Pathological stage } & (2) & 1 & $2.0 \%$ \\
\hline & I & 13 & $25.5 \%$ \\
\hline & II & 15 & $29.4 \%$ \\
\hline & III & 22 & $43.1 \%$ \\
\hline \multirow{5}{*}{ урT stage } & 0 & 1 & $2.0 \%$ \\
\hline & 1 & 10 & $19.6 \%$ \\
\hline & 2 & 9 & $17.6 \%$ \\
\hline & 3 & 25 & $49.0 \%$ \\
\hline & 4 & 6 & $11.8 \%$ \\
\hline \multirow{3}{*}{ ypN stage } & 0 & 30 & $58.8 \%$ \\
\hline & 1 & 18 & $35.3 \%$ \\
\hline & 2 & 3 & $5.9 \%$ \\
\hline \multirow{2}{*}{ Lymphovascular invasion } & $(-)$ & 36 & $70.6 \%$ \\
\hline & $(+)$ & 15 & $29.4 \%$ \\
\hline \multirow{2}{*}{ Perineural invasion } & $(-)$ & 32 & $62.7 \%$ \\
\hline & $(+)$ & 19 & $37.3 \%$ \\
\hline \multirow{2}{*}{ pTRG/AJCC } & $0-1$ & 19 & $37.3 \%$ \\
\hline & $2-3$ & 32 & $62.7 \%$ \\
\hline \multicolumn{4}{|c|}{$\begin{array}{l}\text { LN: Iymph node, Pre-CRT: pre-chemoradiotherapy, Post-CRT: post- } \\
\text { chemoradiotherapy, 3D-CRT: three-dimensional conformal radiotherapy, IMRT: } \\
\text { intensity-modulated radiotherapy, VMAT: volumetric arc therapy, ypT stage: } \\
\text { postoperative pathological T stage, ypN stage: postoperative pathological N } \\
\text { stage, nCRT: neoadjuvant chemoradiotherapy, pTRG/AJCC: pathological tumour } \\
\text { regression grade/American Joint Committee on Cancer (2018-8th edition). }\end{array}$} \\
\hline
\end{tabular}

In this study, the pTRG scoring system of the AJCC was used. A TRG of 0 (pCR) was achieved in 1 of the patients, a TRG of 1 in 18 of the patients, a TRG of 2 in 14 of the patients, and a TRG of 3 in 18 of the patients. TRG 0 and 1 ( $n=19$, $37.3 \%)$ and TRG 2 and $3(n=32,62.7 \%)$ responders were included in the group that did not respond well to nCRT. In our study, the PTRG had no effect in the single or multivariate analyses on either DFS or OS $(p>0.05)$.

In a large series study, 914 LARC patients were examined. Patients were given three different chemotherapy schemes as IV bolus 5-FU, IV infusion 5-FU, and oral capecitabine simultaneously, with 45-50.4 Gy RT. A 25\% pCR was obtained in the IV bolus group, $18 \%$ in the capecitabine group, and $15 \%$ in the IV infusion group, but there was no statistically significant difference. ${ }^{17}$ In this study, patients were given 45 Gy RT concurrently with oral capecitabine. Only one patient had pCR. However, in 5 of the 21 patients who refused surgery after nCRT, MRI showed complete radiological response. Since these patients were excluded from the study, and our series was small, pCR was considered below.
In one study, tumours located in the upper rectum were compared with tumours in the middle-lower position in terms of DFS and OS. PCR, DFS, and OS were found to be better in tumours located in the upper rectum $(p<0.05){ }^{18}$ In this study, no such difference was observed in DFS and OS, but the number of patients with upper-rectal located tumours in this study was only two. In another study, comparisons were made according to the interval between sugery and nCRT, and no difference was observed in terms of local recurrence between $<8$ weeks and $\geq 8$ weeks. ${ }^{19}$ Same was the observation in this study.

Studies have been investigating the effects of $\mathrm{LN}+$ status on DFS and OS. In one of these studies, 130 patients were included, while the number of $\mathrm{LN}+$ patients detected preoperatively was 77 ; this number decreased to 41 postoperatively. While only four of the 37 patients (TRG 1 and 2) who were accepted as 'responders' to neoadjuvant therapy were $\mathrm{N}+$ $(10.8 \%)$, the rest were N0 $(89.2 \%)$. Of the 93 patients who were considered to be 'unresponsive' to neoadjuvant therapy (TRG 3, 4, and 5), 37 patients were $\mathrm{N}+(39.8 \%)$ and 56 patients $(60.2 \%)$ were NO $(p<0.001)$. Based on these results, it was concluded that the response to $\mathrm{nCRT}$ in rectal cancer was associated with LN positivity. ${ }^{20}$ In this study, LN+ was detected in 43 patients (84.3\%) nCRT, while this number decreased to $18(35.3 \%)$ in the post-CRT MRI. The number of $\mathrm{LN}+$ patients detected ypN was 21 (41.2\%) (18 patients N1, 2 patients N1c, 3 patients N2). It is evident in this study that nCRT led to a significant decrease in the number of LN+ patients. A significant effect of post-CRT LN+ status, the ypN stage, and the pLN+ number on DFS was observed. Moreover, in the multivariate reduced model, a significant independent effect of the pLN+ number was observed on DFS time. In another study, the LN number, ratio, and surface area were important prognostic factors in $\mathrm{nCRT} .{ }^{21}$ In another study, the LN regression grade (LRG) was shown to be affecting DFS, and it was suggested that LRG be considered a prognostic marker in patients with rectal cancer as an indicator of their response to $\mathrm{nCRT}{ }^{22}$

There have been several studies that have investigated the effect of the pathological TNM stage, PNI, and LVI on prognosis. In one study, a 5-grade TRG scoring system was used, and the presence of PNI and LVI were examined histologically. Although there was a significant relationship between TRG and survival, no effect of TRG on survival was observed in patients with PNI and LVI. Excluding patients with PNI and LVI, TRG was identified as an independent prognostic factor with regard to DFS and OS. ${ }^{10}$ In another study, LVI was shown to benefit from nCRT significantly, while PNI did not. A multivariate analysis revealed that PNI is an independent prognostic factor for both DFS and OS. PNI is a significant prognostic factor for rectal cancer patients treated with nCRT, especially when a $<8 \mathrm{LN}$ are collected. The lack of effect of nCRT on PNI indicates that residual tumour cells with PNI are more radio-resistant or biologically aggressive than those without. $^{23}$ 
Table III a: Factors influencing DFS (Cox regression analysis).

\begin{tabular}{|c|c|c|c|c|c|c|c|c|c|c|}
\hline \multirow[b]{3}{*}{ Age at diagnosis in years } & \multicolumn{5}{|c|}{ Univariate Model } & \multicolumn{5}{|c|}{ Multivariate Model } \\
\hline & \multirow{2}{*}{$\begin{array}{c}\text { HR } \\
1.03\end{array}$} & \multicolumn{3}{|c|}{$\begin{array}{c}95 \% \text { confidence } \\
\text { interval }\end{array}$} & \multirow{2}{*}{$\begin{array}{c}\boldsymbol{p} \\
0.393\end{array}$} & \multirow[t]{2}{*}{ HR } & \multicolumn{3}{|c|}{$\begin{array}{l}\text { 95\% confidence } \\
\text { interval }\end{array}$} & \multirow[t]{2}{*}{$\boldsymbol{p}$} \\
\hline & & 0.96 & - & 1.10 & & & & & & \\
\hline Gender & 0.81 & 0.23 & - & 2.80 & 0.739 & & & & & \\
\hline Location of the tumour & 1.96 & 0.71 & - & 5.50 & 0.196 & & & & & \\
\hline Radiological LN+ (pre-CRT) & 1.74 & 0.22 & - & 13.76 & 0.599 & & & & & \\
\hline Radiological LN+ (post-CRT) & 4.65 & 1.20 & - & 18.0 & 0.026 & & & & & \\
\hline $\begin{array}{l}\text { RT technique: } \\
\text { IMRT } \\
\text { VMAT }\end{array}$ & $\begin{array}{l}1.82 \\
5.50 \\
1.93\end{array}$ & $\begin{array}{l}0.85 \\
1.42 \\
0.21\end{array}$ & $\begin{array}{l}- \\
- \\
-\end{array}$ & $\begin{array}{l}3.88 \\
21.3 \\
17.4\end{array}$ & $\begin{array}{l}0.123 \\
\mathbf{0 . 0 1 3} \\
0.558\end{array}$ & $\begin{array}{l}10.4 \\
7.57\end{array}$ & $\begin{array}{l}2.02 \\
0.55\end{array}$ & $\begin{array}{l}- \\
-\end{array}$ & $\begin{array}{c}54.02 \\
103.68\end{array}$ & $\begin{array}{l}\mathbf{0 . 0 0 5} \\
0.130\end{array}$ \\
\hline Time between diagnosis and nCRT & 1.13 & 0.33 & - & 3.92 & 0.845 & & & & & \\
\hline Time between nCRT and surgery & 0.98 & 0.28 & - & 3.40 & 0.971 & & & & & \\
\hline Histopathology & 0.72 & 0.09 & - & 5.66 & 0.752 & & & & & \\
\hline Pat tumour diameter after nCRT & 1.04 & 1.01 & - & 1.08 & 0.006 & & & & & \\
\hline Pathological stage & 4.30 & 1.14 & - & 16.19 & 0.031 & & & & & \\
\hline ypT stage & 3.81 & 1.46 & - & 9.92 & 0.006 & 9.58 & 1.77 & - & 52.0 & 0.009 \\
\hline ypN stage & 2.75 & 1.12 & - & 6.75 & 0.027 & & & & & \\
\hline pLN+ number & 1.24 & 1.05 & - & 1.46 & 0.011 & 1.39 & 1.11 & - & 1.74 & 0.004 \\
\hline LVI & 3.80 & 1.07 & - & 13.46 & 0.039 & & & & & \\
\hline PNI & 2.93 & 0.83 & - & 10.41 & 0.096 & & & & & \\
\hline pTRG/AJCC & 1.49 & 0.38 & - & 5.78 & 0.566 & & & & & \\
\hline
\end{tabular}

Table III b: Factors influencing OS (Cox regression analysis).

\begin{tabular}{|c|c|c|c|c|c|c|c|c|c|c|}
\hline \multirow[b]{3}{*}{ Age at diagnosis in years } & \multicolumn{5}{|c|}{ Univariate Model } & \multicolumn{5}{|c|}{ Multivariate Model } \\
\hline & \multirow{2}{*}{$\begin{array}{c}\text { HR } \\
1.05\end{array}$} & \multicolumn{3}{|c|}{ 95\% confidence interval } & \multirow{2}{*}{$\begin{array}{c}\boldsymbol{p} \\
0.276\end{array}$} & \multirow[t]{2}{*}{ HR } & \multicolumn{3}{|c|}{ 95\% confidence interval } & \multirow[t]{2}{*}{$p$} \\
\hline & & 0.96 & - & 1.15 & & & & & & \\
\hline Gender & 0.80 & 0.16 & - & 3.99 & 0.790 & & & & & \\
\hline Location of the tumour & 0.76 & 0.18 & - & 3.21 & 0.710 & & & & & \\
\hline Radiological LN+ (pre-CRT) & 25.78 & 0.00 & - & $>100$ & 0.551 & & & & & \\
\hline Radiological LN+ (post-CRT) & 1.55 & 0.31 & - & 7.74 & 0.594 & & & & & \\
\hline RT technique & 1.33 & 0.43 & - & 4.09 & 0.614 & & & & & \\
\hline Time between diagnosis and nCRT & 0.54 & 0.10 & - & 2.97 & 0.478 & & & & & \\
\hline Time between nCRT and surgery & 6.99 & 0.76 & - & 63.87 & 0.085 & & & & & \\
\hline Histopathology & 1.33 & 0.16 & - & 11.41 & 0.794 & & & & & \\
\hline Pat tumour diameter after nCRT & 1.04 & 1.00 & - & 1.09 & 0.049 & & & & & \\
\hline Pathological stage & 4.55 & 0.70 & - & 29.75 & 0.113 & & & & & \\
\hline ypT stage & 4.59 & 1.12 & - & 18.85 & 0.034 & & & & & \\
\hline ypN stage & 2.01 & 0.66 & - & 6.17 & 0.221 & & & & & \\
\hline $\mathrm{pLN}+$ number & 1.16 & 0.97 & - & 1.39 & 0.102 & & & & & \\
\hline LVI & 4.69 & 0.86 & - & 25.62 & 0.074 & & & & & \\
\hline PNI & 9.35 & 1.09 & - & 80.27 & 0.041 & & & & & \\
\hline pTRG/AJCC & 3.14 & 0.37 & - & 26.96 & 0.296 & & & & & \\
\hline Recurrence & 20.67 & 2.41 & - & 177.02 & 0.006 & 20.67 & 2.41 & - & 177.02 & 0.006 \\
\hline
\end{tabular}




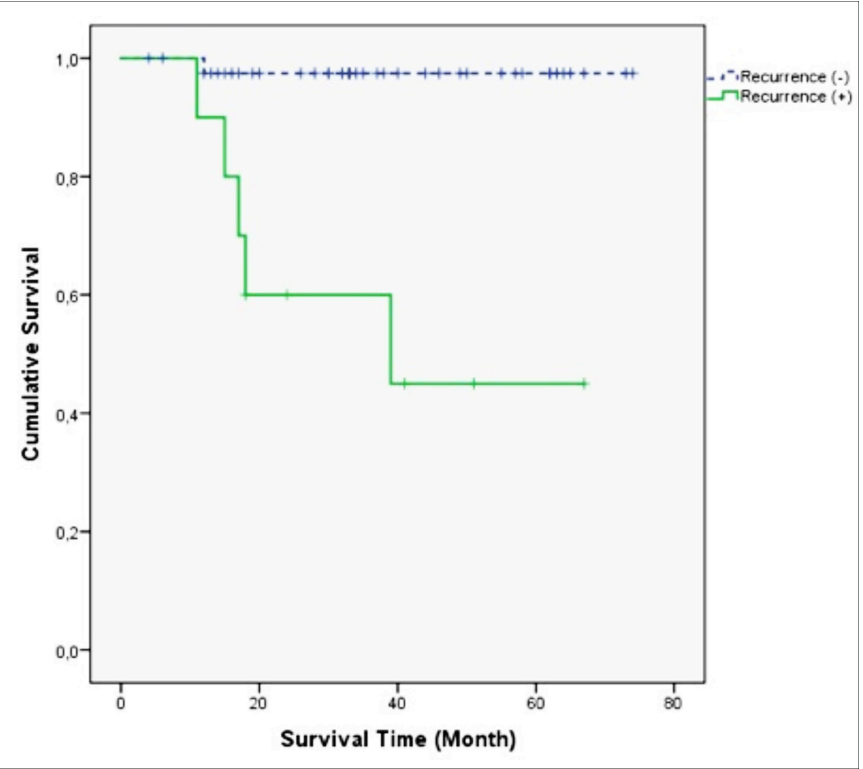

Figure 2: The effect of recurrence on OS (Kaplan-Meier).

Similarly, in another study, a low ypT, ypN, and pTNM, as well as LVI/PNI absence, were associated with better DFS and OS in a univariate analysis. In a multivariate analysis, ypT and LVI were identified as independent prognostic factors for DFS and $\mathrm{OS}^{24}$ In a study where more factors were investigated, when the factors affecting DFS were analysed, neural, vascular, and lymphatic invasion, pre-and postoperative CEA, TNM staging, smoking, and the histological response to nCRT were found to be significant. As for OS, TNM staging, the histological grade, peritumoral inflammatory reaction, and vascular and neural invasion were found to have a significant effect. ${ }^{25}$

In this study, LVI significantly affected DFS, in the univariate analysis, but no effect on OS was observed. While PNI was not effective for DFS in the univariate analysis, it showed a significant effect on OS. The ypT stage had significant effect for DFS and OS in the univariate analysis, while it had a significant independent effect on DFS in the multivariate analysis. The predicted DFS in the group with ypT stage 4 was significantly shorter than that of the group with ypT stage 3. Similarly, in the univariate analysis, the effect of pathological tumour diameter after nCRT on DFS and OS was observed. The predicted DFS in the group with a diameter of $\leq 2 \mathrm{~cm}$ was significantly longer than that of the group with a tumour diameter of $>2 \mathrm{~cm}$.

\section{CONCLUSION}

Many factors affect the prognosis of LARC patients who have received $n C R T$. While the effect of the PTRG on survival was not observed in the study, the post-chemoradiotherapy radiological LN+, pathological tumour diameter after nCRT, LVI, pathological stage, ypT, ypN, and pLN+ number affected DFS, and the pathological tumour diameter after nCRT, ypT stage, and PNI had a significant effect on OS. The predicted
DFS time as the pathological tumour diameter after nCRT and ypT stage increased, as well as the predicted OS time as recurrence increased, were shortened.

\section{ETHICAL APPROVAL:}

All procedures performed in studies involving human participants were by the institutional and/or national research committee's ethical standards; and the 1964 Helsinki Declaration and its later amendments or comparable ethical standards. Institutional Review Board approval was obtained for this study. The study was conducted with the Non-interventional Clinical Research Ethics Committee of Erciyes University Faculty of Medicine's approval. (Ethic's Committee approval No. 2020/62).

\section{CONFLICT OF INTEREST:}

The authors declared no conflict of interest.

\section{AUTHORS' CONTRIBUTION:}

AA: Concept design, material and data collection, analysis and interpretation, literature review and writing the study, and critical review.

EA: Concept and design, data collection, analysis and literature review.

GT: Supervision of the study, material and data collection.

SKE: Supervision of the study, fundings related to the study and literature review.

All authors had full access to the data in the study and took responsibility for the data integrity and data analysis, accuracy.

\section{REFERENCES}

1. Bipat S, Glas AS, Slors FJM, Zwinderman AH, Bossuyt PMM, Stoker J. Rectal cancer: Local staging and assessment of lymph node involvement with endoluminal US, CT, and MR Imaging - a meta-analysis. Radiology 2004; 232(3):773-83. doi: 10.1148/radiol.2323031368.

2. Minsky BD, Rödel CM, Valentini V. Rectal Cancer. Clinical Radiation Oncol 2016; 51: 992-1018.

3. Rullier E, Denost Q, Vendrely V, Rullier A, Laurent C. Low rectal cancer: classification and standardization of surgery. Dis Colon Rectum. 2013; 56(5): 560-7. doi: 10.1097/DCR. 0b013e31827c4a8c.

4. Habr-Gama A, Perez RO. Non-operative management of rectal cancer after neoadjuvant chemoradiation. Br J Surg 2009; 96(2): 125-7. doi: 10.1002/bjs.6470.

5. Dhadda AS, Dickinson P, Zaitoun AM, Gandhi N, Bessell EM. Prognostic importance of Mandard tumour regression grade following pre-operative chemo/radiotherapy for locally advanced rectal cancer. Eur J Cancer 2011; 47(8):1138-45. doi: 10.1016/j.ejca.2010.12.006.

6. Fokas E, Liersch T, Fietkau R, Hohenberger W, Beissbarth T, Hess $C$, et al. Tumor regression grading after pre-operative chemoradiotherapy for locally advanced rectal carcinoma revisited: Updated results of the CAO/ARO/AIO-94 trial. J Clin Oncol 2014; 32(15):1554-62. doi: 10.1200/JCO.2013. 54.3769 . 
7. AJCC Cancer Stagıng Manual, 8th Edition. 2018.

8. Arredondo J, Baixauli J, Beorlegui C, Arbea L, Rodríguez J, Sola J, et al. Prognosis factors for recurrence in patients with locally advanced rectal cancer preoperatively treated with chemoradiotherapy and adjuvant chemotherapy. Dis Colon Rectum 2013; 56(4):416-21. doi: 10.1097/DCR.0b013 e318274d9c6.

9. Wei J, Huang R, Guo S, Zhang X, Xi S, Wang Q, et al. ypTNM category combined with AJCC tumor regression grade for screening patients with the worst prognosis after neoadjuvant chemoradiation therapy for locally advanced rectal cancer. Cancer Manag Res 2018; 10:5219-25. doi: 10.2147/ CMAR.S179151.

10. Cienfuegos JA, Rotellar F, Baixauli J, Beorlegui C, Sola $\mathrm{J}$, Arbea L, et al. Impact of perineural and lymphovascular invasion on oncological outcomes in rectal cancer treated with neoadjuvant chemoradiotherapy and surgery. Ann Surg Oncol 2015; 22(3):916-23. doi: 10.1245/s10434-0144051-5.

11. Sauer R, Becker H, Hohenberger P. Pre-operative chemoradiotherapy as compared with postoperative chemoradiotherapy for locally advanced rectal cancer. New Engl J Med 2004; 351: 11-20.

12. Dworak O, Keilholz L, Hoffmann A. Pathological features of rectal cancer after pre-operative radiochemotherapy. Int J Colorectal Dis 1997; 12:19-23.

13. Park YJ, Oh BR, Lim SW, Huh JW, Joo JK, Kim YJ, et al. Clinical significance of tumor regression grade in rectal cancer with pre-operative chemoradiotherapy. J Korean Soc Coloproctol. 2010; 26:279-86. doi: 10.3393/jksc.2010. 26.4.279.

14. Rodel C, Martus P, Papadoupolos T. Prognostic significant for tumor regression after preopative chemoradiotherapy for rectal cancer. J Clin Oncol 2005; 23: 8688-96.

15. Mandard AM, Dalibard F, Mandard JC, Marnay J, Amar MH, Petiot JF, et al. Pathologic assessment of tumor regression after pre-operative chemoradiotherapy of esophageal carcinoma. Clinicopathologic correlations. Cancer. 1994; 73: 2680-6. doi: 10.1002/1097-0142(19940601).

16. Trakarnsanga A, Gönen M, Shia J, Nash GM, Temple LK. Guillem JC, et al. Comparison of tumor regression grade systems for locally advanced rectal cancer after multimodality treatment. J Natl Cancer Inst 2014; 22:106(10). pii: dju248. doi: 10.1093/jnci/dju248.

17. Kılıc D, Sert F, Gorken IB, Alicikus ZA, Akturk N, Sağlam EK, et al. Prognostic significance of early complete response in patients with locally advanced rectal cancer undergoing preoperative chemoradiotherapy: Multicentric Study of Turkish Society for Radiation Oncology Group (TROD). Turk J Gastroenterol 2020; 31(5):368-77. doi: 10.5152/tjg.2020. 19225.

18. Huang MY, Lee HH, Tsai HL, Huang CW, Yeh YS, Ma Cl, et al. Comparison of efficacy and safety of pre-operative chemoradiotherapy in locally advanced upper and middle/lower rectal cancer. Radiat Oncol 2018; 13(1):53. doi: 10.1186/ s13014-018-0987-0.

19. Mihmanlı M, Kabul Gürbulak E, Akgün IE, Celayir MF, Yazıcı $P$, Tunçel $D$, et al. Delaying surgery after neoadjuvant chemoradiotherapy improves prognosis of rectal cancer. World J Gastrointest Oncol. 2016 Sep 15; 8(9):695-706. doi: 10.4251/wjgo.v8.i9.695.

20. Garcia-Florez LJ, Gomez-Alvarez G, Frunza AM, Barneo-Serra $L$, Fresno Forcelledo MF. Response to chemoradiotherapy and lymph node involvement in locally advanced rectal cancer. World J Gastrointest Surg 2015; 7(9):196-202. doi: 10.4240/wjgs.v7.i9.196.

21. Pitto F, Zoppoli G, Scabini S, Romairone E, Fiocca R, Ballestrero $A$, et al. Lymph node number, surface area and lymph node ratio are important prognostic indicators in neoadjuvant chemoradiotherapy treated rectal cancer. J Clin Pathol 2020; 73(3):162-6. doi: 10.1136/jclinpath-2019206139.

22. Choi JP, Kim SJ, Park IJ, Hong SM, Lee JL, Yoon YS, et al. IS the pathological regression level of metastatic lymph nodes associated with oncologic outcomes following pre-operative chemoradiotherapy in rectal cancer? Oncotarget 2017; 8(6):10375-84. doi: 10.18632/oncotarget.14418.

23. Kim CH, Yeom SS, Lee SY, Kim HR, Kim YJ, Lee KH, et al. Prognostic Impact of perineural Invasion in rectal cancer after neoadjuvant chemoradiotherapy. World J Surg 2019; 43(1):260-72. doi: 10.1007/s00268-018-4774-8.

24. Cho HJ, Baek JH, Baek DW, Kang BW, Lee SJ, Kim HJ, et al. Prognostic significance of clinicopathological and molecular features after neoadjuvant chemoradiotherapy in rectal cancer patients. In Vivo 2019; 33(6):1959-65. doi: 10.21873/invivo.11691.

25. Moral M, Fdez-Aceñero MJ, Cuberes R, Suárez A. Factors influencing prognosis after neo-adjuvant chemoradiation therapy for rectal carcinoma. Acta Chir Belg 2009; 109(3): 345-51. doi: 10.1080/00015458.2009.11680437. 